\title{
VITRUVIO INTERPRETADO POR UN ARQUITECTO DE NUEVA ESPAÑA EN EL SIGLO XVII
}

\author{
P O R
}

\author{
M A N U L T O US A I N T
}

\begin{abstract}
Gon tan escasas las referencias documentales acerca de la técnica que D siguieron los"arquitectos coloniales en México, que cualquier referencia, sobre todo si viene de ellos mismos, ofrece interés. Conocemos las ordenanzas del gfemio, unas del siglo xvi rudimentarias y otras del xvirr en las que, comô símbolo del progreso, de lo que se trata es de favorecer a los agremiados: para la menor reparación de edificios, salvo tapar goteras o blanquear muros, debería llamarse a un arquitecto. Exactamente lo mismo que, salvo un dolor de cabeza o un catarro, que es lo único que podemos curarnos por nuestra propia mano, para cualquier otra enfermedad por leve que sea hay que llamar a un médico.

La mayor parte de los arquitectos coloniales estaba constituida por hombres prácticos, de habilidad mantual, de mucha experiencia, pero ignorantes. Ignorantes no ya de los tratadistas clásicos de su arte que se reducian a Vitruvio, Serlio, León Bautista Alberti, Viñola y escasamente Paladio, sino de los elementos de la geometría. Alarifes existieron que no
\end{abstract}


sabían escribir y desempeñaron su cargo a satisfacción. $Y$ así se llega al extremo que censura este arquitecto de que hoy hablamos y ridiculiza más tarde, en forma graciosísima, Tresguerras.

Mas no todo era incultura en los maestros edificadores de Nueva España. Poseemos, y hemos dado a conocer, las listas de 105 libros de arquitectura que llegaban a México (gracias al celo del Santo Oficio). Conocemos arquitectos, como Melchor Pérez de Soto que poseía una biblioteca que para sus tiempos, primer tercio del siglo xvII, era estupenda, si bien contaminada con muchos tratados de astrología como la de su colega el agrimensor alemán Henrico Martínez.

No cabe dudar que los arquitectos de nuestras grandes catedrales, Alcaraz, Arziniega, Agüero, Becerra, los Gómez de Trasmonte, García Ferrer, Casillas, Pérez de Castañeda, Ortiz de Castro, Tolsá y tantos otros poseían tanto la teoría como la práctica, pero no debe olvidarse que González Velázquez y Costanzó truenan, en las postrimerías de la colonia, contra la ignorancia de los arquitectos.

Rodrigo Díaz de Aguilera desempeñaba en 1668 el cargo de "Maestro arquitecto, Aparejador maior de la obra y fábrica de la santa iglesia catedral de México". Había adquirido un ejemplar, acaso el más interesante, de la obra de Vitruvio. Se trata de la edición quizás más rara de este libro. El colofón reza: "Argentorati (Estrasburgo), ex of ficina Knobbechiana, per Georgium Machaeropieum, mense augusto Anno MDL."”

El bibliógrafo Brunet no cita en su Manual sino ediciones posteriores. El libro se ve enriquecido con las anotaciones de Guillermo Filandro y apéndices de Julio Frontino y Nicolás Cusano. Lleva índices de los vocablos griegos con la correspondencia latina.

Maese Rodrigo entendía bien su latín, pero además le interesaba mucho su oficio. Por eso escribe anotaciones de su puño y letra en las márgenes del libro. No son tantas como deseáramos, mas nos indican un anhelo, una ansia de comprender el texto del maestro y sus anotadores, y a veces aparecen observaciones personales acerca de México que las avaloran más aún. Las reproducimos como existen, sean ya meras traducciones fragmentarias o anotaciones suyas. Su valor es él mismo.

Lib. I. Cap. I. La arquitectura consta de teórica y práctica. Teórica o especulativa es la que por hallar la causa de los efectos, de la práctica considera la proporción como una especulación del en- 
tendimiento. Según definición de Euclides, la práctica pone en execusión las razones que el entendimiento especuló en la teórica.

Los que se contentaron con sólo la teórica, sin la experiencia de la práctica, sólo han conseguido la sombra de la arquitectura. $\mathrm{Y}$ sólo se puede llamar arquitecto el que, en lo teórico y práctico ha llegado a ser perfecto.

¿ Pues cómo se podrá llamar arquitecto quién no sabe apenas echar una firma? Este no se estendería más de a ser un oficial de albañil.

Graphis llama, a las lineas con que se desen ... las plantas y monteas para explicar con más claridad la forma del edificio. -Anotaciones. - Cariátides llama unas estatuas de mujeres en lugar de columnas sobre sus plintos y recibiendo los capiteles.

Cap. II. De que cosas consta la arquitectura, esenciales y forzosas de saber el arquitecto.

Ordenación es una pequeña medida sacada con proporción de todas las proporciones en simetría del todo con sus partes.

Quantidad es el dicho módulo conveniente con el todo y sus partes.

Ignografía, quiere decir la planta que se delínea en que se reparte todos los compartimientos del edificio.

Ortografía es lo que llamamos montea que muestra la fachada del edificio con sus repartimientos de puertas y ventanas en todos sus miembros.

Scenografía en las anotaciones entiende lo mismo que ortografía.

Es simétría una ordenación en las partes, con tal disposición que cada una corresponda con el todo y el todo con sus partes.

Lib. Ir. Cap. IIr. Pág. 63: De 4 Géneros de arena será la mejor la que cogida en la mano y refregándola y apretándola hiciere ruido, o echándola en un paño blanco y refregándola no quedare señal de tierra.

Cap. vi. Estas arenas que aquí pone Vitruvio can las calidades que expresa es propiamente el tesontlali que usamos en esta ciudad de México. Léase con cuidado todo este capítulo.

Lib. III. Prefacio. Los artífices que con el trabajo de su estudio adquirieron ciencia, adquirieron por la ciencia la nobleza que no tuvieron por su nacimiento. 
Muchas veces son obscurecidas las virtudes de bueno y docto artífice por la ignorancia de los que lo juzgan.

Cap. Ir. Anotaciones. Lugar citado dice León Baptista: 5 géneros se quentan de intervalos los quales llamaremos asi, esparcido, espeso, elegante, menos esparcido, menos espeso. Prosigue desde el $N^{\circ} 15$, los espacios de entre las colunas han de ser nones y las colunas han de ser pares y en los intervalos anchos usa de las colunas más gruesas así que las grosesas se medirán por los intervalos y los intervalos por las colunas siguiendo estas leies que en las obras espesas los intervalos tengan de grueço no menos que grosesa y media de coluna, pero en las que hubieren de ser más esparcidas tendrá no más que añidiendo también tres de las 8 partes de una coluna, en las elegantes tendrá dos grosesas y una quarta parte de una. En las menos esparcidas se darán 3 enteras grosesas, y en las menos espesas dos, pero en los intervalos que están medios en los órdenes, estos se harán más anchos que los otros, de modo que los exceda en una quarta parte suia.

Lib. IIr. Cap. IIr. A esto llaman fugere dificultaten. Esta es la razón porque algunos antiguos dixeron no ser la obra dórica para elegiria para templos por las dificultades de repartir los triglifos y metopas.

Tetrástylos es y quiere decir de quatro colunas, y aunque dice que la frente para este orden se parta en 27 partes parece estar errado, y Sebastiano Serlio en el libro 4 que trata de la orden doria a fojas 22 trata deste texto y la distribución destas columnaciones, en sus espacios altos.

Di noticia de este libro y sus anotaciones en 1927, en el tomo vi de la obra Iglesias de México. Recogió la información el Marqués de Lozoya en su Historia del Arte Hispánico. Por eso creo interesante reproducir ahora las anotaciones como testimonio espiritual de un arquitecto de Nueva España, de plena época barroca, lo que viene a demostrar que a través de todos los tiempos, de todas las modalidades porque pasó 'Ia arquitectura del mundo, después de Grecia, subsiste o informa, real o disfrazada, motivo o pretexto, la ordenación clásica. 
DOI: http://dx.doi.org/10.22201/iie.18703062e.1950.18.494

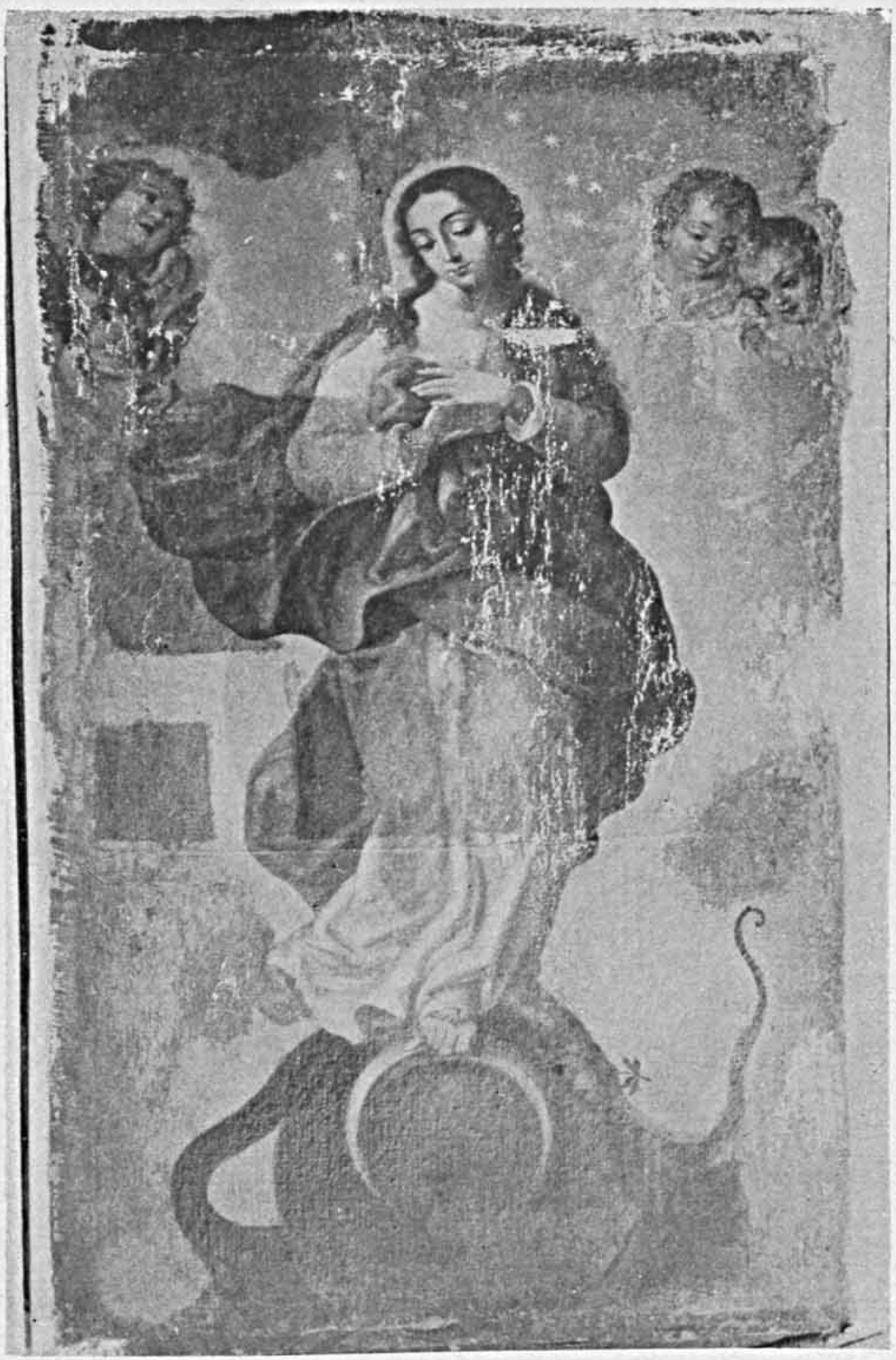

1. Purísima. Por Mariano Morelos. 1806 
DOI: http://dx.doi.org/10.22201/iie.18703062e.1950.18.494

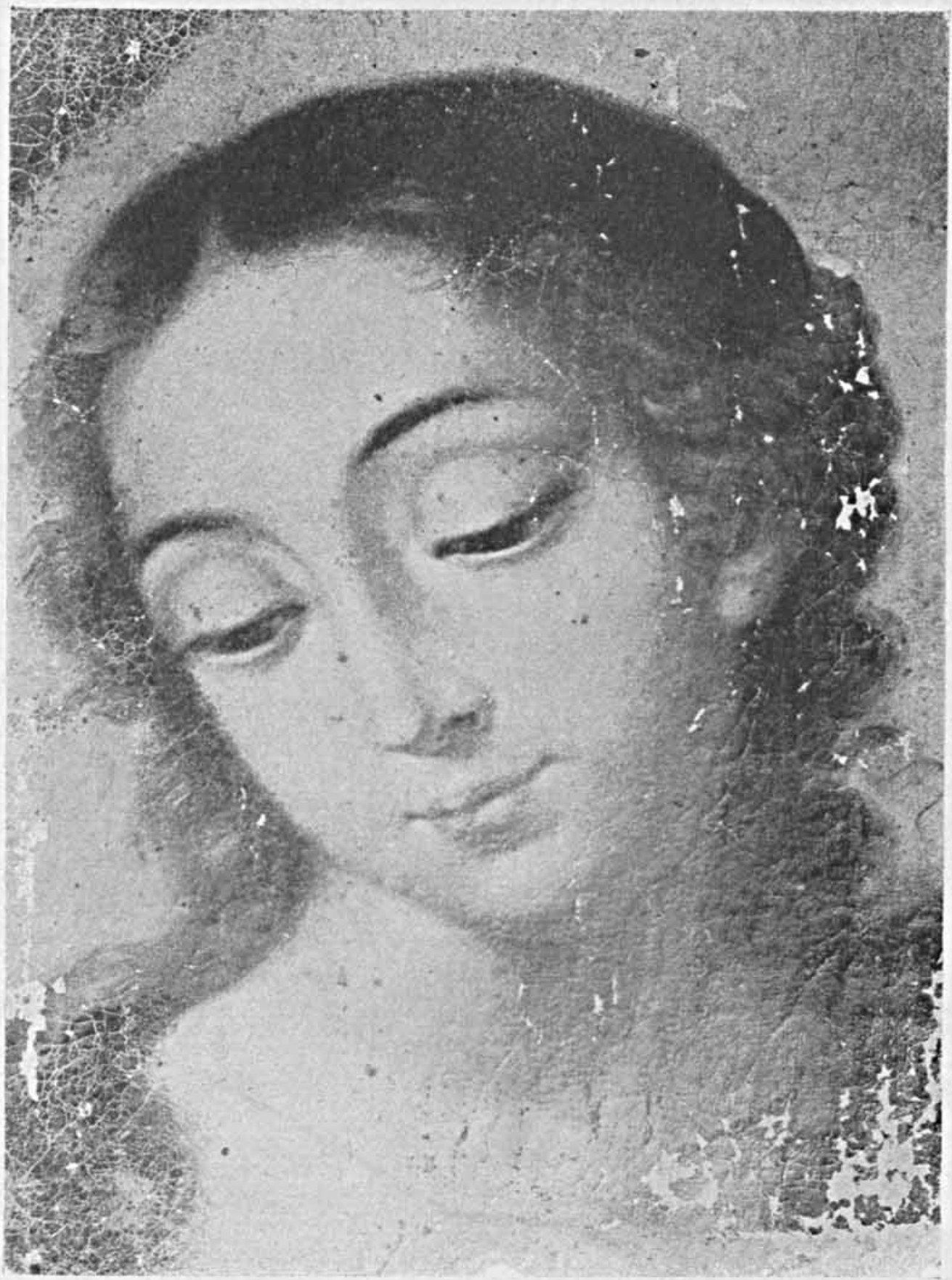

2. Purisima. Detalle 
DOI: http://dx.doi.org/10.22201/iie.18703062e.1950.18.494

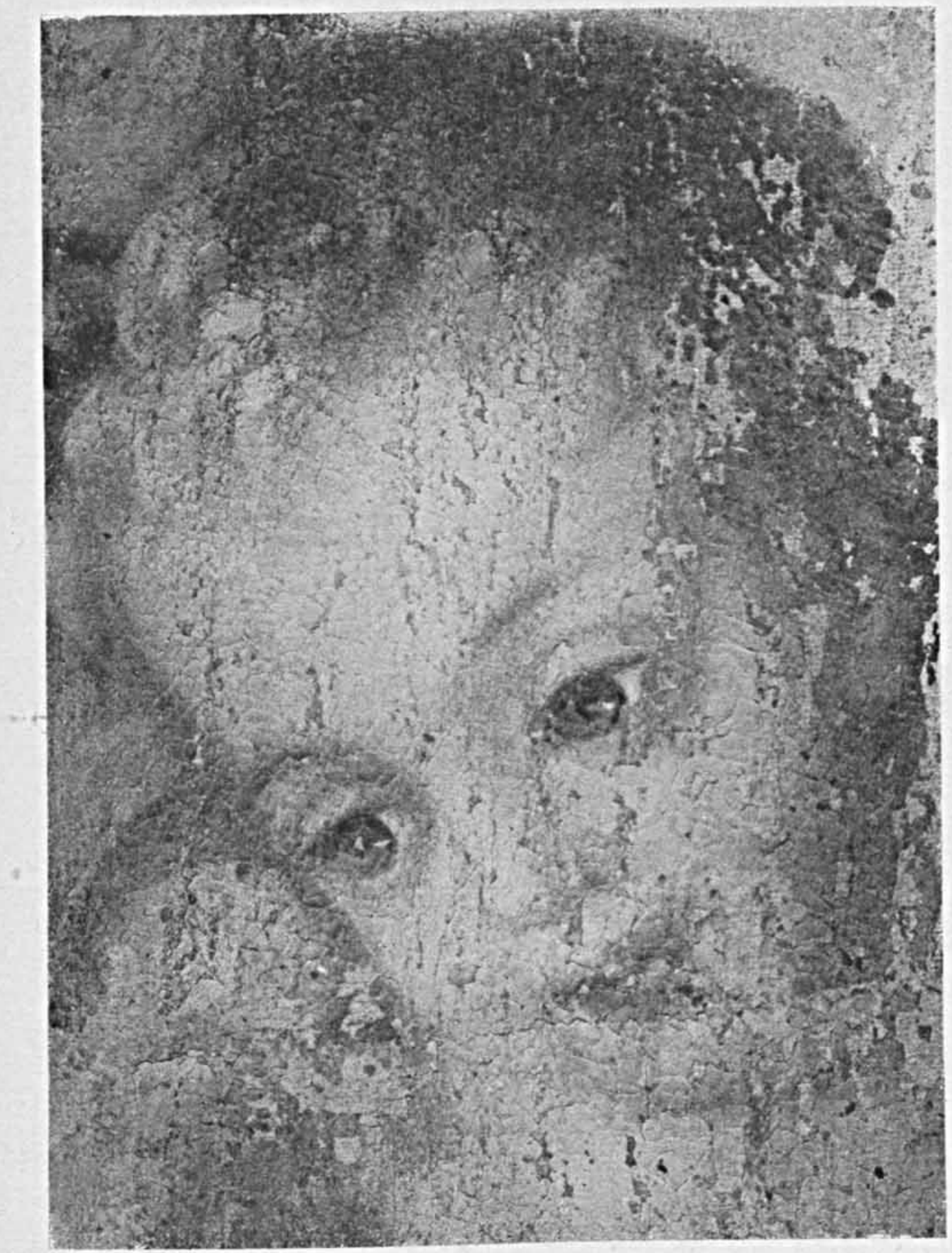

3. Detalle del querubín de la derecha 
DOI: http://dx.doi.org/10.22201/iie.18703062e.1950.18.494

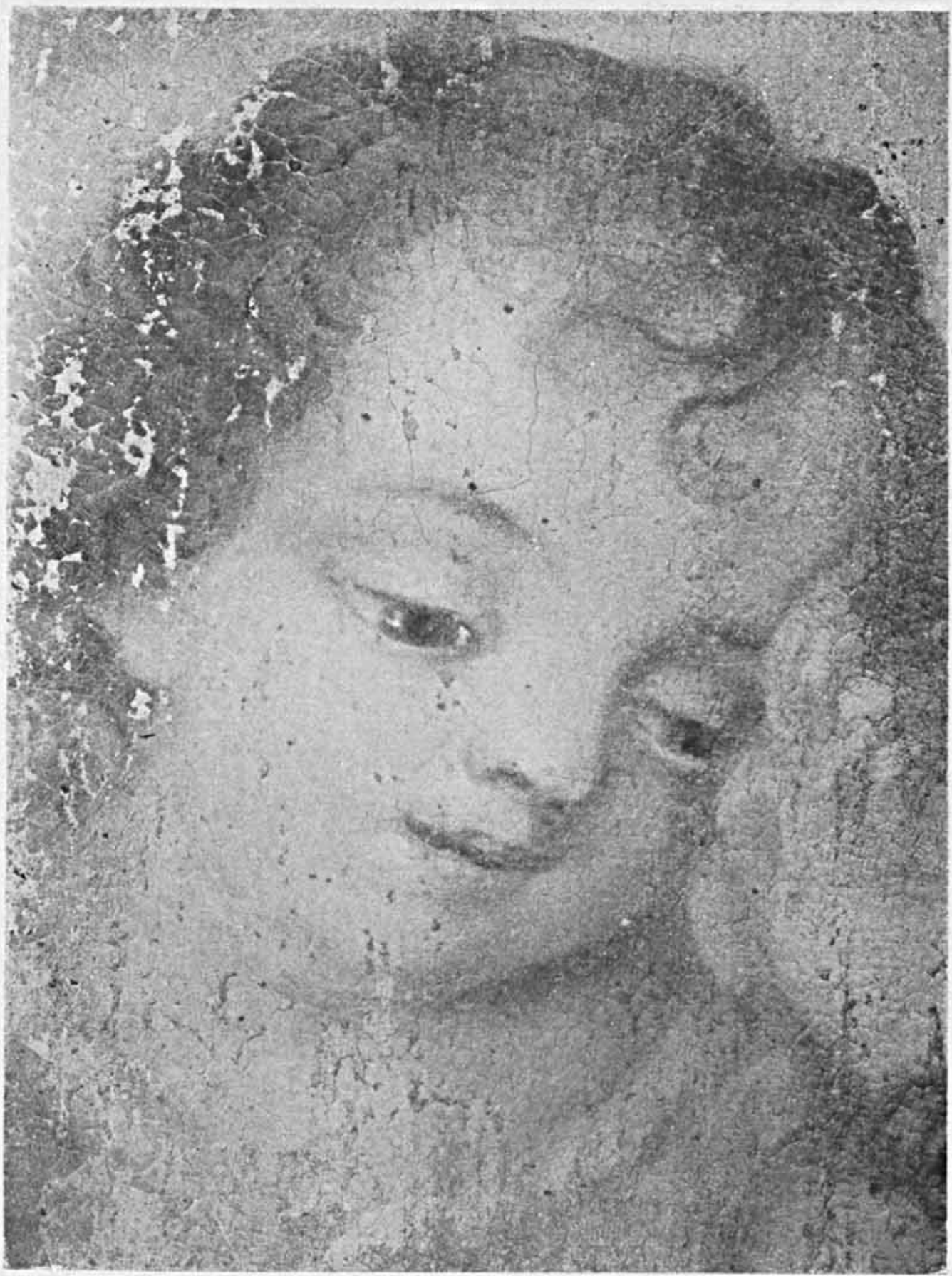

4. Detalle del otro querubín de la derecha 